\title{
A Drosophila Orthologue of Larp Protein Family Is Required for Multiple Processes in Male Meiosis
}

\author{
Keiko Ichihara $^{1,2}$, Hanako Shimizu ${ }^{1,2}$, Osamu Taguchi ${ }^{3}$, Masamitsu Yamaguchi ${ }^{2,4}$ \\ and Yoshihiro H. Inoue $\mathrm{e}^{12^{*}}$ \\ ${ }^{1}$ Drosophila Genetic Resource Center, Kyoto Institute of Technology, Kyoto 616-8354, Japan, ${ }^{2}$ Insect \\ Biomedical Research Center, Kyoto Institute of Technology, Kyoto 616-8354, Japan, ${ }^{3}$ Aichi Cancer Center \\ Research Institute, Nagoya 464-8681, Japan, and ${ }^{4}$ Department of Applied Biology, Kyoto Institute of \\ Technology, Kyoto 616-8354, Japan
}

\begin{abstract}
$A B S T R A C T$. It is important for the proper execution of cell division in both mitosis and meiosis that the chromosome segregation, cytokinesis, and partition of cell organelles progress in smooth coordination. We show here that the mitochondria inheritance is closely linked with microtubules during meiotic divisions in Drosophila males. They are first clustered in a cell equator at metaphase associated with astral microtubules and then distributed along central spindle microtubules after anaphase. The molecular mechanism for the microtubuledependent inheritance of mitochondria in male meiosis has not been demonstrated yet. We first isolated mutations for a larp gene that is highly conserved among eukaryotes and showed that these mutant males exhibited multiple meiotic phenotypes such as a failure of chromosome segregation, cytokinesis, and mitochondrial partition. Our cytological examination revealed that the mutants showed defects in spindle pole organization and spindle formation. The larp encodes a Drosophila orthologue of a La-related protein containing a domain exhibiting an outstanding homology with a La type RNA-binding protein. Surprisingly, the dLarp protein is localized in the cytoplasm of the male germ line cells, as observed by its distinct co-localization with mitochondria in early spermatocytes and during meiotic divisions. We discuss here the essential role that dLarp plays in multiple processes in Drosophila male meiosis.
\end{abstract}

Key words: Drosophila/meiosis/spindle microtubules/mitochondrial inheritance/Larp

\section{Introduction}

Cell division is principally composed of two consecutive processes, chromosome segregation and cytokinesis. Many studies have pointed out that a considerable fraction of indispensable proteins for chromosome segregation or cytokinesis are closely linked with microtubules with regard to their function and localization (for review, Vernos and Karsenti, 1998; Eggert et al., 2006). While a general view of the mechanisms that control chromosome segregation or cytokinesis has been progressively uncovered, the inherit-

\footnotetext{
*To whom correspondence should be addressed: Yoshihiro H. Inoue, Drosophila Genetic Resource Center, Kyoto Institute of Technology, Sagaippongi-cho, Ukyo-ku, Kyoto 616-8354, Japan.

Tel: +81-75-873-2653, Fax: +81-75-861-0881

E-mail: yhinoue@kit.ac.jp

Abbreviations: Larp, La-related protein; La, Lupas antigen; CNN, centrosomin; DAPI, 4'6-diamidino-2-phenylindole; MnSOD, manganese superoxide dismutase; GFP, green fluorescence protein; PAGE, polyacrylamide gel electrophoresis; ORF, open reading frame.
}

ance of cytoplasmic components remains relatively poorly understood. It is important for successful cell division that these cellular events progress in smooth coordination with each other. It had been believed that the inheritance of welldispersed cytoplasmic organelles such as mitochondria is achieved passively as a consequence of equal cytokinesis (Shima and Warren, 1998). However, recent studies have implicated that the mitochondria repeat fission and fusion in a dynamic fashion in living cells (Kiefel et al., 2006). Their active transport along cytoplasmic microtubules driven by a kinesin-related protein, KIF1B, has also been characterized in neuronal cells (Nangaku et al., 1994). In fission yeast, the spindle microtubules indeed mediate positioning and inheritance of mitochondria (Yaffe et al., 2003). However, it is largely unknown whether active cellular mechanisms to ensure equal inheritance of the mitochondria exist in animal cells.

Drosophila male meiosis provides us with some advantages in the precise examination of chromosome segre- 
gation and organelle partition (Fuller, 1993). After a completion of meiotic divisions, the mitochondria in each spermatid make a single large aggregate, the so-called Nebenkern. Every spermatid in a cyst at onion stage contains equally-sized nucleus and Nebenkern. This is achieved as a consequence of proper chromosome segregation and cytokinesis as well as equal partition of mitochondria. This provides us with a quite convenient and sensitive system to find cell division defects simultaneously in living spermatids (Castrillon et al., 1993). Previous electron microscopy studies by Tates in 1971 have described that mitochondria translocate to and assemble near the cell division plane in male meiosis (reviewed in Fuller, 1993). However, the details of mitochondrial distribution as a meiotic progression remain to be examined using current techniques. Several mutants for genes required for spindle organization exhibit not only defects in chromosome segregation and cytokinesis during male meiosis but also showed a perturbation of mitochondria inheritance (Fuller et al., 1988; Casal et al., 1990; Bonaccorsi et al., 1998). These genetic data allowed us to carry out a further examination of the interaction between mitochondria and microtubules.

As a consequence of a large-scale genetic screening, we have isolated new allelic mutations that disrupted the cell cycle progression. In this paper, we describe meiotic phenotypes of mutants for the larp gene that encodes a Drosophila orthologue of an uncharacterized protein family that is widely conserved. It has a conserved domain homologous to a La type RNA-binding protein. The larp mutant males exhibited abnormal segregation of chromatids and a failure of cytokinesis in male meiosis. In addition, they indicate perturbations of the mitochondrial inheritance. We first describe that the mitochondria inheritance is closely associated with astral or central spindle microtubules according to meiotic stages. Surprisingly, the dLarp exhibited a distinctive co-localization with mitochondria during meiotic divisions or at earlier spermatocyte stages. In this paper, we discuss the role of the dLarp required for the proper progression of Drosophila male meiosis. These results give us valuable information for understanding the indispensable function of the conserved Larp family.

\section{Materials and Methods}

\section{Fly strains}

We isolated new allelic mutations, $\operatorname{larp}^{m t r l}$ and $\operatorname{larp}^{\text {mtrw }}$ among a subset of a collection of P element-mediated mutations (Deak et al., 1997; Inoue et al., 2000). In situ hybridization revealed that both mutants carried a placw insertion on $98 \mathrm{C} 3$ region. A genetic mapping of male sterile mutation on larp $^{\text {mtrw }}$ using 8 visible markers placed it at 3-95.7, which is closely linked to the insertion site. Another allele, larp $^{m t r}$ was obtained as a mutation by a $P Z$ insertion on $98 \mathrm{C} 3$. The original chromosome for $\operatorname{larp}^{m t r 2}$ carried a lethal mutation, l(3)06487 separable from larp ${ }^{m t r 2}$. More than thirty $w$ or $r y$ progenies were respectively obtained among progenies from dysgenic males carrying $\Delta 2-3$. Most of such revertants were phenotypic revertants for male and female sterility. A larp ${ }^{43}$ carrying a PiggyBac insertion at 98C3 (Horn et al., 2003) failed to complement other larp mutations. A reversion analysis of the integrated PiggyBac also confirmed that it was responsible for their sterility. Another null type allele, larp ${ }^{\text {null } \# 4}$, that was a gift from Dr. Melanie Gatt of Cambridge University, is lacking whole coding exons.

\section{Cytological examination of living testes}

To examine the morphology of spermatids at onion stage in living material, testes from pharate adults were dissected and gently squashed under a coverslip. The live preparations were immediately observed by a phase contrast microscopy (Inoue et al., 2004).

\section{dLarp antibody and Western blot analysis}

A $1.3 \mathrm{~kb}$ long cDNA fragment for the larp was inserted into expression vectors to express a fusion protein of its polypeptide corresponding to 16-444 amino acids with the carboxy terminus of glutathione S-transferase (GST) protein. Antiserum was prepared by injecting the purified GST-dLarp into a rat as described (Inoue et al., 2000). We affinity-purified antibodies specific to the dLarp from antiserum with GST-dLarp conjugated Sepharose. For Western blot analysis, approximately equal volumes of adult testes were collected from wild-type or larp mutants. Protein extracts from each genotype were used for electrophoresis and were transferred to a PVDF membrane. The blots were incubated with the affinitypurified antibody diluted 1:500.

\section{Immunocytochemistry}

Immunostaining of testes was carried out as previously described (Inoue et al., 2004). The affinity-purified anti-dLarp IgG was diluted 1:200 for immunostaining. Microtubules were detected by expression of GFP tagged $\alpha$ Tubulin (Inoue et al., 2004) or by immunostaining using anti-Tubulin antibody. Centrosomes were revealed with anti-CNN antibody (Li and Kaufman, 1995). Mitochondria were visualized with MitoFluor Red (Molecular Probe) as described elsewhere (Arama et al., 2006) or by immunostaining with anti-MnSOD antibody (Stressgen). Alternatively, mitochondria were detected with fluorescence from EYFP tagged with mitochondria targeting sequences in $s q$ h-EYFP-Mito strain. A protein trap line ZCL2496, in which a trap vector was integrated into the first intron of the larp upstream to coding exons, was used to examine a localization of GFP-dLarp. All preparations were observed with fluorescence microscope equipped with a cooled charge-coupled device camera. The developmental stage of primary spermatocytes at growth phase was determined as described (Cenci et al., 1994). 


\section{Results}

\section{A sterile complementation group, meteor (mtr), that is required for a cell cycle progression of germ line cells, corresponds to a conserved gene encoding a Drosophila La-related protein}

In order to isolate novel genes required for a cell cycle progression, we performed a genetic screening among a subset of a collection of $P$ element-induced mutations and isolated new allelic mutations, $m t r^{l}$ and $m t r^{w}$ that exhibited sterility in both males and females. We obtained another allele, $m t r^{2}$ induced by $P Z$ insertion. In early stage embryos from females homozygous for these mutations, a perturbation of cell cycle progression or nuclear division was frequently observed. These $m t r$ mutants also possessed smaller testes that led us to suspect a defect in cell proliferation or differentiation during spermatogenesis. All mtr alleles were responsible for $\mathrm{P}$ element insertions on 98C3. DNA sequencing of those flanking genomic sequences revealed that every mutation had a single insertion in the first intron of the CG14066 gene, also known as larp. A ubiquitous expression of a $4.6 \mathrm{~kb}$ long cDNA corresponding a predicted larp-RB transcript of the CG14066 rescued a sterility of $m t^{w}$ in both females and males. These data indicated that a gene responsible for the mtr mutations corresponded to the CG14066/larp. We, therefore, designated $m t r^{w}, m t^{l}$ and $m t r^{2}$ as $l a r p^{m t r w}, \operatorname{larp}^{m t r l}$ and $l a r p^{m t r 2}$, respectively. As another allele, larp ${ }^{43}$ encodes a truncated protein lacking its carboxy-terminal half due to a PiggyBac insertion in a coding exon (Fig. 5A), it was regarded as a null allele. The $\operatorname{larp}^{43}$ flies were viable but male- and female-sterile. Both homozygotes and transheterozygotes between larp mutations exhibited normal morphology and viability except for shorter and thinner macrocheates.

\section{The larp mutant males show multiple defects in meiotic divisions such as chromosome segregation, cytokinesis and mitochondria partition}

The maternal phenotypes and a loss of male fertility of larp mutants allowed us to further examine if they showed defects in male meiosis. We first examined living spermatids at onion stage under a phase contrast microscope. In wild type, every spermatid at post-meiotic onion stage contained single nucleus and a Nebenkern (Fig. 1A, Table I). The nucleus and the Nebenkern are uniform in size among every spermatid. These post-meiotic figures indicated that chromosome segregation, partition of mitochondria and cytokinesis were properly executed in meiotic divisions (Fuller, 1993). On the other hand, an average of $42.0 \%$ of spermatids from larp ${ }^{m t r w}$ males showed a variety of abnor-
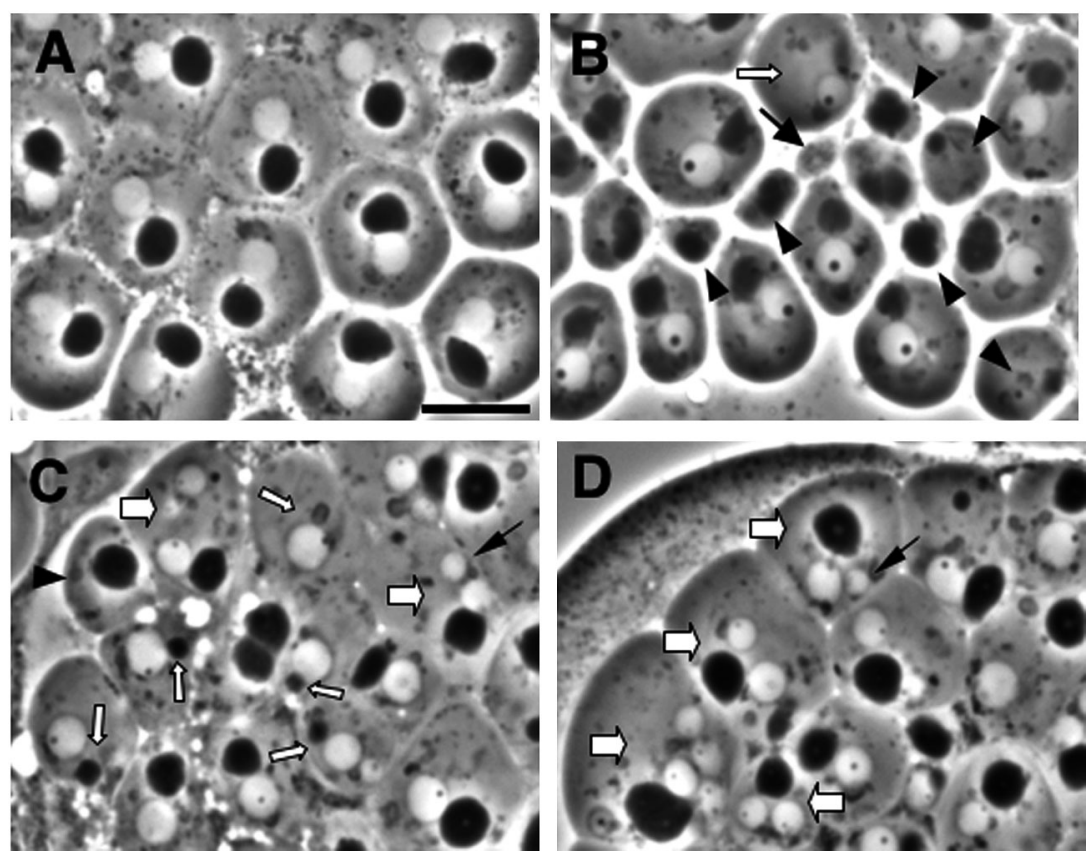

Fig. 1. Defects in chromosome segregation, cytokinesis and mitochondria partition during meiotic divisions in larp mutant males. (A) A phase-contrast micrograph of a living spermatid cyst at onion stage from wild type. Note that the presence of a single light nucleus and a single dark Nebenkern equal in size in each spermatid. (B-D) Spermatids at onion stage from larp ${ }^{m t r w}$ males. Arrowheads show smaller cells without nuclei (arrowheads). Thinner arrows in $\mathrm{C}$ and $\mathrm{D}$ indicate smaller nuclei in size. Cells pointed by smaller white arrows contain smaller or no Nebenkerns. An arrow in B indicates a cell without any nuclei or Nebenkerns. Larger white arrows in $\mathrm{C}$ and $\mathrm{D}$ point cells containing more than two nuclei, indicating a failure of cytokinesis. Bar indicates $10 \mu \mathrm{m}$. 
Table I. MULTIPLE PHENOTYPES IN SPERMATIDS AT ONION STAGE FROM LARP MUTANT MALES

\begin{tabular}{lcccccccc}
\hline genotype & $\begin{array}{c}\text { total \#s of } \\
\text { spermatids at } \\
\text { onion stage }\end{array}$ & cysts & $\begin{array}{c}\text { total \#s of } \\
\text { normal } \\
\text { cells }(\%)\end{array}$ & $\begin{array}{c}\text { total \#s of } \\
\text { abnormal } \\
\text { cells }(\%)\end{array}$ & $\begin{array}{c}\text { abnormal } \\
\text { cell \#s } \\
\text { or size }\end{array}$ & $\begin{array}{c}\text { abnormal } \\
\text { Nebenkern \#s } \\
\text { or size }\end{array}$ & $\begin{array}{c}\text { a failure of } \\
\text { cytokinesis }\end{array}$ & $\begin{array}{c}\text { cells with } \\
\text { multiple } \\
\text { abnormalities }\end{array}$ \\
\hline Canton S (wild-type) & 865 & 18 & $860(99.5)$ & $5(0.5)$ & $0(0)$ & $0(0)$ & $5(100.0)$ & $0(0)$ \\
Larp & 530 & 13 & $212(40.0)$ & $318(60.0)$ & $198(49.3)$ & $160(39.8)$ & $44(10.9)$ & $84(26.4)$ \\
larp $^{\text {mtrw }}$ & 450 & 10 & $261(58.0)$ & $189(42.0)$ & $100(38.3)$ & $91(34.9)$ & $70(26.8)$ & $72(38.1)$ \\
larp $^{\text {mtrl } / \text { larp }}$ & 379 & 8 & $260(68.6)$ & $119(31.4)$ & $64(52.0)$ & $43(35.0)$ & $16(13.0)$ & $4(3.4)$ \\
\hline
\end{tabular}

a) The living spermatids at post meiotic onion stage from wild-type (Canton $S$ ) and larp mutant males were examined a number and size of nucleus or Nebenkern, aggregate of mitochondria in each cell.

malities (Table I, Fig. 1B-D), and $38.8 \%$ of the abnormal spermatids possessed various-sized nuclei. A considerable fraction of the abnormal spermatids contained smaller-sized nuclei (Fig. 1, thinner arrows), which were similar to the abnormal figures observed commonly among mutants for spindle formation (Fuller et al., 1988; Inoue et al., 2004). Spermatids without nuclei were also scored in the mutants (Fig. 1, arrowheads). This type of spermatid would be generated from a defect in chromosome segregation during meiotic divisions. In addition, $26.8 \%$ of the abnormal spermatids from the mutants contained a single Nebenkern associated with multiple nuclei with normal size suggesting a failure of cytokinesis during one or both of the meiotic divisions (Fig. 1, larger white arrows). Furthermore, another $34.9 \%$ of abnormal spermatids from the mutant cysts had variously sized, mainly smaller, Nebenkerns (Fig. 1, smaller white arrows). Some abnormal spermatids in this category contained multiple smaller pieces of Nebenkerns. It was characteristic of the larp mutants that they exhibited not only chromosome segregation defects but also showed defects in mitochondria partition. Even spermatids lacking both nuclei and Nebenkerns were observed in the mutant cysts (Fig. 1B, arrow). A considerable fraction of abnormal spermatids (38.1\%) exhibited composite abnormalities such as a combination of defects in chromosome segregation and in cytokinesis in the larp mutant. Those multiple meiotic phenotypes can be also seen in other homozygotes or every allelic combination for four larp mutations (Table I).

\section{The larp mutants show several defects in spindle pole organization and spindle formation at meiotic divisions in males}

In order to understand the common source of defects in chromosome segregation and cytokinesis, we examined the meiotic apparatuses from the larp mutants. By a comparison with normal meiotic cells, in which bipolar spindle structure was intrinsically associated with asters at both poles (inset in Fig. 2A), several abnormalities in pole organization or its function were observed in the larp mutants. First, the mutants frequently produced abnormal cells in which a subset of spindle microtubules was not connected to spindle poles at metaphase. They failed to orient along the spindle axis defined by the two asters (Fig. 2A). Second, we also found that extra foci of centrosome antigen were contained in some cells within metaphase I cysts from the mutant (Fig. $2 \mathrm{~B}$, open arrow). In addition to a loss of centrosome integrity, microtubules around spindle poles appeared not to be focused on the spindle poles in such cells (Fig. 2B, filled arrow). Spindle pole organization and/or pole function as microtubule-organizing center were thus perturbed in the mutant meiotic cells.

Further, cells containing four centrosomes, as a consequence of a failure of previous cytokinesis, have been frequently scored in meiosis II cyst from larp mutants. We found that the central spindle structure essential for cytokinesis was not formed in some telophase cells, in which cleavage furrow ingression had not taken place (open arrow in Fig. 2D). Such a defect in central spindle formation leads to a failure of cytokinesis as shown in Fig. 1.

In addition, we have sometimes observed abnormal meiotic figures in which a chromosome mass was dislocated from a cytoplasmic space between poles in the mutants (Fig. $2 \mathrm{C}$, open arrow). Although a cleavage furrow ingression progressed in telophase cells, it was also observed that chromosomes had not separated and a chromosome mass was contained in only one daughter cell (data not shown). It seems to be likely that such abnormal cells would result in a generation of anucleate cells as shown in Fig. 2C. Another possible interpretation for the failure of chromosome segregation would be related to a failure of chromosome capture.

\section{A mitochondria distribution along aster and spindle microtubules in male meiotic divisions}

A perturbation of mitochondria distribution in the mutants allowed us to perform detailed examination of mitochondria inheritance with microtubules in normal meiotic progression. At the beginning of prophase I, mitochondria were homogenously distributed throughout the cytoplasm includ- 

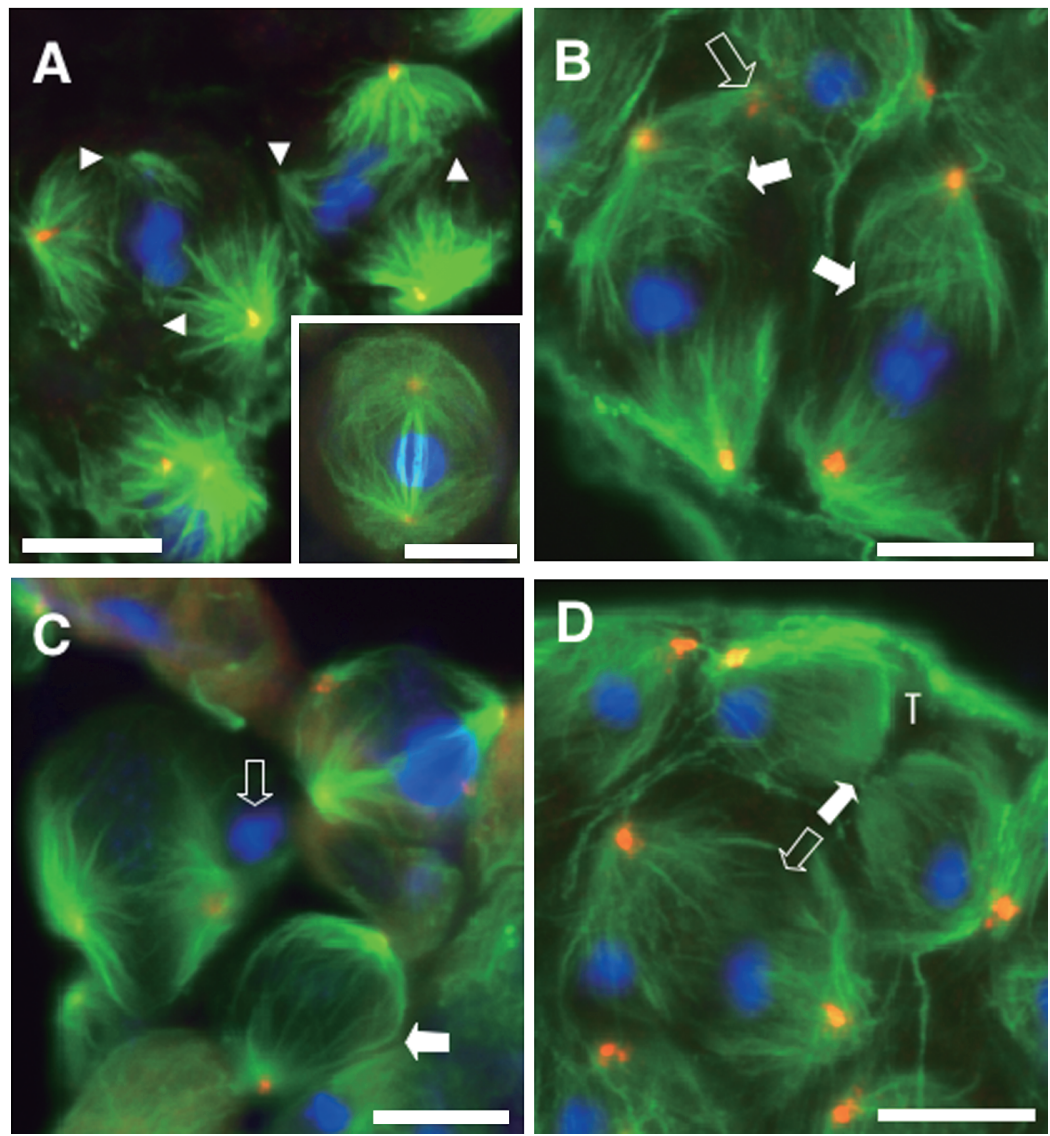

Fig. 2. Abnormal spindle structure and a loss of spindle pole integrity in meiotic cells from larp mutant males. (A) Subsets of spindle microtubules are detached from both spindle poles and fail to orient along spindle axis in metaphase I cells from larp ${ }^{m t r w}$, while all spindle microtubules are tightly anchored on spindle poles in wild-type (inset). Arrowheads point minus ends of the spindle microtubules. (B) A part of centrosome is disintegrated from a spindle pole (open arrow) and microtubules around upper poles are not adequately focused (filled arrows). (C) A chromosome mass is dislocated between two spindle poles (open arrow) in metaphase I cells from larp ${ }^{m t r w}$, suggesting a failure of chromosome capture. A spermatocyte at metaphase I contains no chromosomes (filled arrow). (D) A loss of central spindle structure is observed in a telophase I cell without ingression of cleavage furrow (open arrow), while robust central spindles are distinct in a normal looking telophase cell (an upper cell labeled as T) with cleavage furrow progression (filled arrow). Microtubules and chromosomes are visualized in green and blue, respectively. Centrosomes are visualized here in yellow due to overlap with anti-tubulin and anti-centrosomin immunostaining. Bars: $10 \mu \mathrm{m}$.

ing the areas occupied by asters (Fig. 3A). As aster microtubules began developing at prophase, mitochondria were expelled from inside of the asters and assembled toward the plus ends of aster microtubules (Fig. 3B). At metaphase, the mitochondria were clustered at the equator of a peripheral cytoplasmic region between two facing asters (Fig. 3C). Such a clustering of mitochondria seems to be dependent on aster microtubules. Then, as central spindle microtubules were formed between separating sister chromatids at anaphase, these mitochondria translocated to the interior region of the cells (Fig. 3D). They distributed along central spindles as if to decorate the microtubule structure (Fig. $3 \mathrm{E})$. Mitochondria along the microtubules dispersed into the cytoplasm, as the central spindles disintegrated to the remnant midbody microtubules (Fig. 3F). At the end of meiotic division II, the mitochondria seemed to be released from the minus end of the midbody microtubules and assembled again to form Nebenkern at onion stage. Thus, the proper segregation of mitochondria in male meiosis is closely associated with distinctive microtubule structures.

\section{Abnormal segregation of mitochondria as well as chromatids during meiotic divisions in the larp mutant males}

In order to understand the primary defect behind the various numbers and sizes of Nebenkerns in the mutants, we examined mitochondria distribution during meiotic division. By a comparison with normal meiotic cells, the mitochondria distribution was significantly affected in the larp males. First, mitochondria failed to be transported along aster microtubules toward their plus ends or failed to assemble at equator 

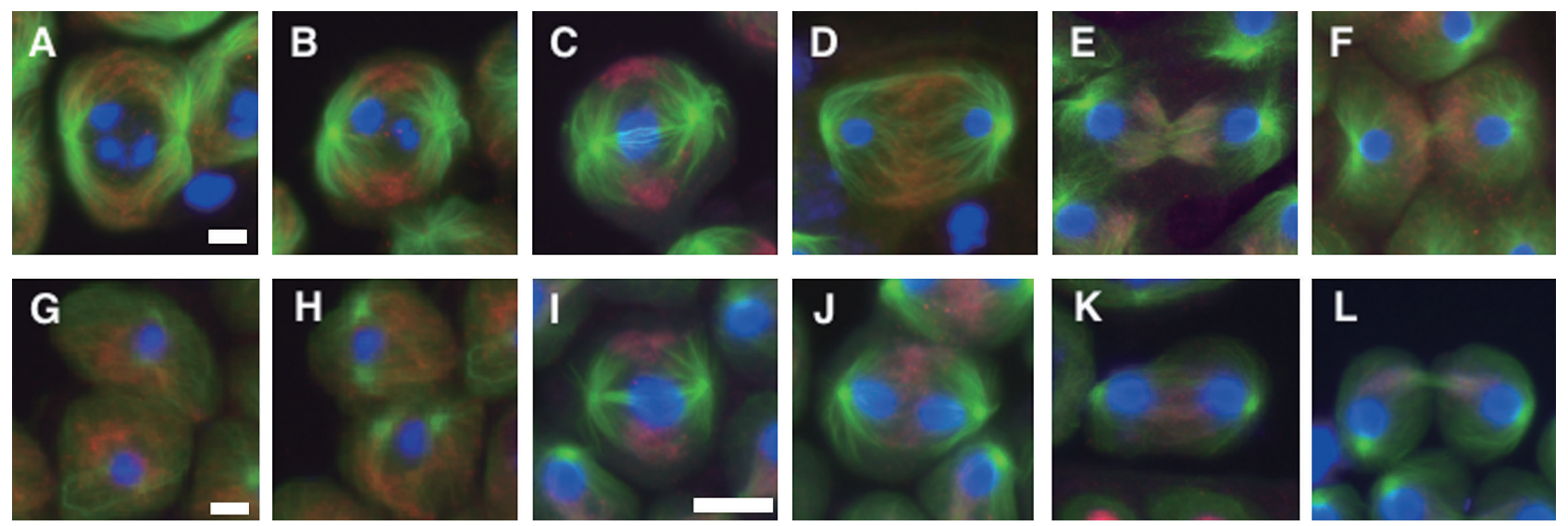

Fig. 3. Distribution of mitochondria is closely correlated with aster and spindle microtubules during meiotic divisions in males. Normal spermatocytes undergoing meiosis I (A-F) or II (G-L) were stained to visualize microtubules (green), mitochondria (red) and chromosomes (blue). Prophase (A, H), prometaphase $(B)$, metaphase $(\mathrm{C}, \mathrm{I})$, anaphase A $(\mathrm{D}, \mathrm{J})$, anaphase B $(\mathrm{E}, \mathrm{K})$, telophase $(\mathrm{F}, \mathrm{L})$ and interphase $(\mathrm{G})$, respectively. Note that homogeneous distribution of mitochondria in cytoplasm at prophase $(\mathrm{A}, \mathrm{H})$. The mitochondria are being expelled around asters (B) and are clustering at equator of peripheral region of metaphase cells $(\mathrm{C}, \mathrm{I})$. Then, they will move into the interior region of the cells, in which central spindle microtubules are assembled at anaphase B (E, K). Bars indicate $10 \mu \mathrm{m}$.
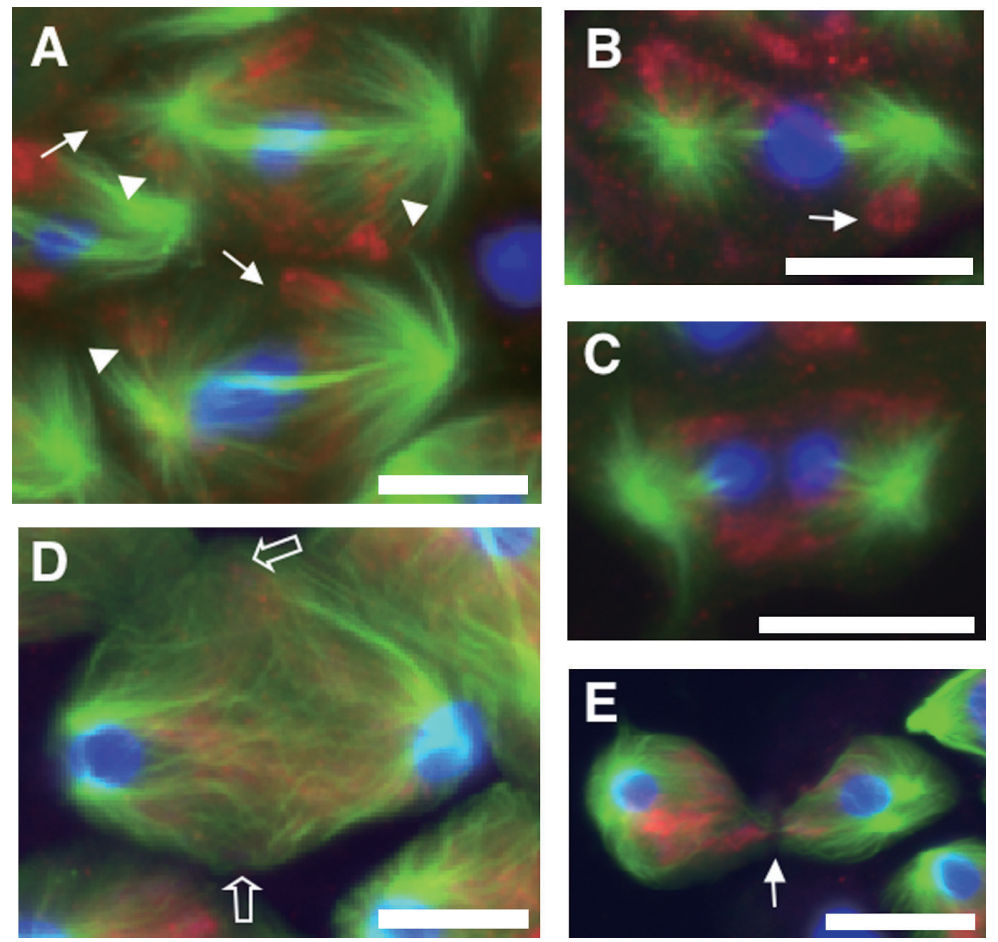

Fig. 4. A mislocalization of mitochondria during meiotic divisions in larp mutant males. (A) Mitochondria have not assembled at equator of peripheral region in metaphase I cells from larp ${ }^{43}$. A considerable fraction of the mitochondria remains present along aster microtubules (arrowheads), while some of the mitochondria continue to remain at the plus end of aster microtubules (arrows). (B) Asymmetric distribution of mitochondria in larp ${ }^{43}$ spermatocytes at metaphase II. Note that the mitochondria continue to cluster at the plus end of aster microtubules (arrow) at one side of these cells. (C) Mitochondria continue to remain along a peripheral region of the cell and have failed to migrate toward the interior in an anaphase II cell from larp ${ }^{m t r w}$. (D) Mitochondria are not restricted to distribution along the central spindles in a telophase I cell without cleavage furrow ingression (open arrows) from larp ${ }^{\text {mtrw }}$. Note that the centrosomes have already separated for a preparation of meiosis II in this telophase I cell. (E) Uneven partition of mitochondria in a late telophase I cell undergoing cytokinesis from larp ${ }^{\text {trtw }}$. An arrow indicates the position of a contractile ring. Microtubules, mitochondria and chromosomes are in green, red and blue, respectively. Bars indicate $10 \mu \mathrm{m}$. 


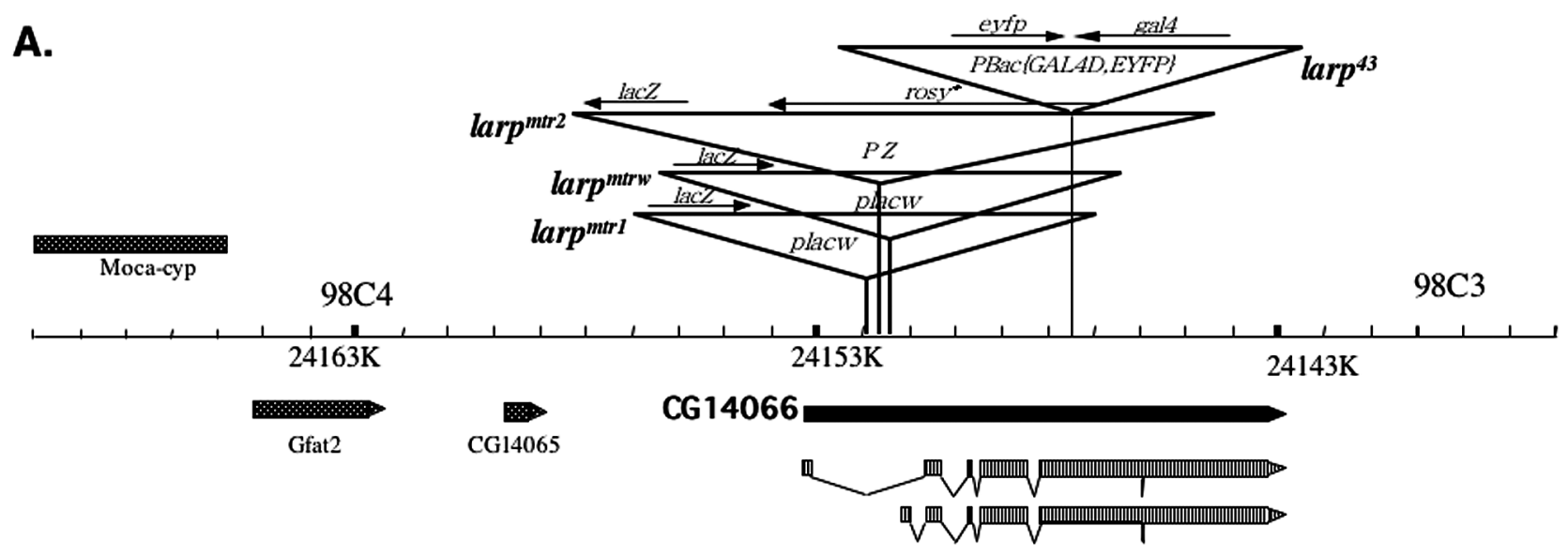

B.

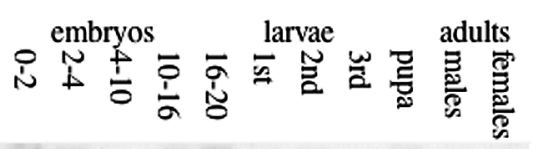

C.
Human
h-Larp

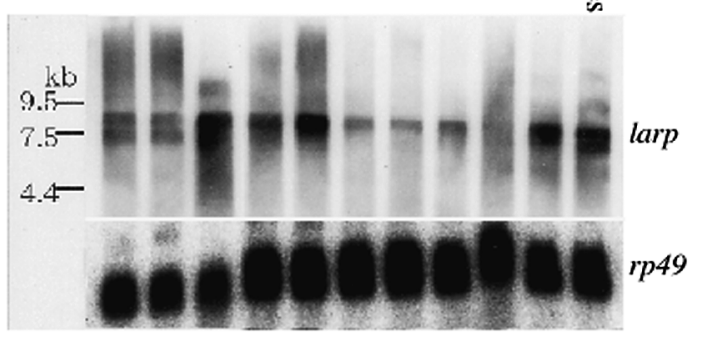

dLarp

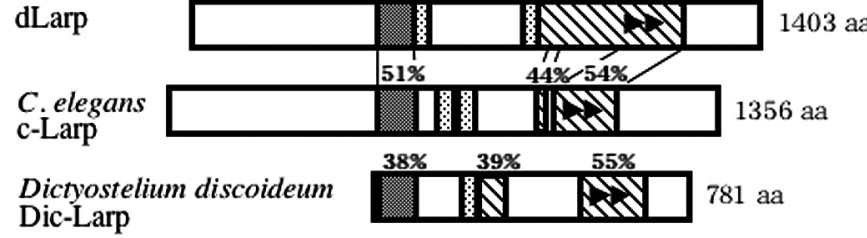

Fig. 5. P element insertions in the larp gene and schematic representation of the larp gene product and its orthologues. (A) A genomic structure of the larp gene and the P element insertions in the larp mutants. (B) Developmental Northern hybridization. (C) A schematic representation of dLarp and its orthologues. The La domain (residues 453 to 544), the conserved domain 1 (residues 818 to 1014) and 2 (residues 1015 to 1250) of the dLarp are marked as LA, C1, and C2, respectively. Short fragments rich in acidic residues are indicated as in small letter. Identities of amino acid sequences between the dLarp and its orthologues are indicated above every domain of the orthologues. Tandem repeats of the DM15 motif are designated by arrowheads.

of metaphase cells (Fig. 4A, B). Second, they had not moved into an interior region at anaphase, in which the central spindle structure was intrinsically constructed (Fig. 4C). The presence of such abnormal cells strongly suggests that the larp is required for proper segregation of mitochondria. Coordinated progress between chromosome segregation, mitochondria partition and cytokinesis was sometimes disrupted in the larp mutants (Fig. 4E).

\section{The larp encodes a member of an evolutionarily conserved protein family with a domain homologous to a La type RNA-binding protein}

The larp gene expressed as a 8.5 kb-long-transcript throughout development and a maternal $7.5 \mathrm{~kb}$-long transcript appeared in early stage embryos as well as female adults (Fig. 5B). The sequences of the longest larp cDNA overlapped with a larp-RB transcript that appeared in the Flybase and revealed it to encode a dLarp protein of 1,403 amino acids (AAN14138). The Blast search predicted that the dLarp contained a La domain lying between residues 453 and 544 homologous to a La RNA-binding protein (Maraia and Intine, 2001) and two tandems repeats of DM15 motif of unknown function (Ponting et al., 2001) at C-terminal region. Another characteristic of the dLarp sequences is that one can find stretches of single amino acids such as poly-glutamine and poly-threonine. Our antibody raised against its $\mathrm{N}$-terminal region recognized a single polypeptide larger than $200 \mathrm{kDa}$ in size in wild-type testis extracts on SDS-PAGE (Fig. 6A). We further raised another two antibodies against each of the 300 amino-acidlong polypeptide from the central region or C-terminal region, respectively. Both antibodies also recognized the same polypeptide band. This band could no longer be detected in testis extracts from null type mutants. It is thus likely that the $\sim 200 \mathrm{kDa}$ polypeptide corresponds to the dLarp. The BLAST searches predicted its orthologues from human (hLarp, BAA34451), mouse (mLarp, XP910122), nematode (T16754) and even in Arabidopsis (NP568409), although no significant orthologues were found in yeast. 

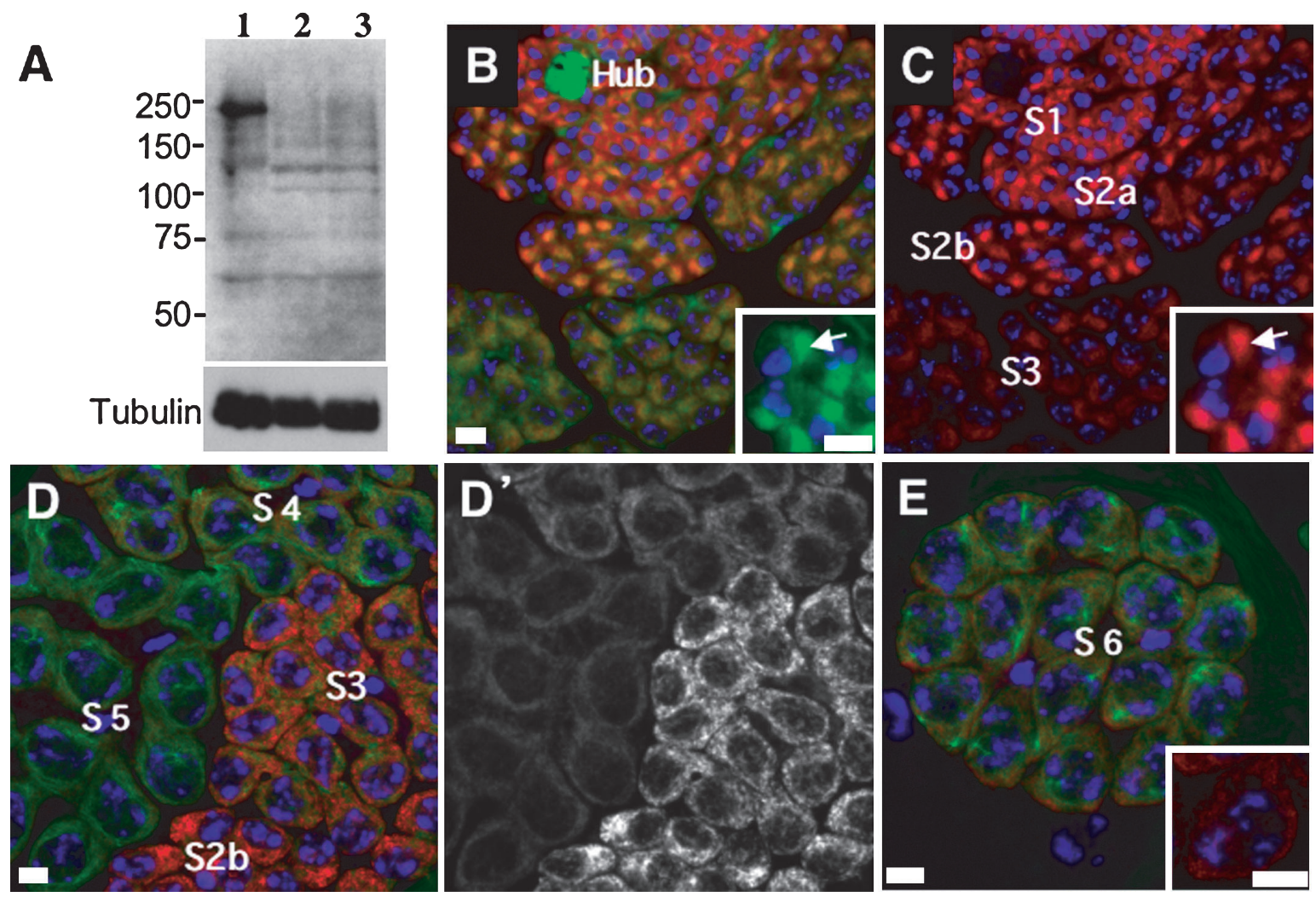

Fig. 6. Immunostaining of testis cells with anti-dLarp antibody. (A) Western blot of testis extracts. The dLarp antibody recognizes a $\sim 200 \mathrm{KDa}$ single polypeptide band (lane 1) that cannot be detected in testis extracts from larp null mutants (larp ${ }^{43}$ (lane 2) or larp ${ }^{\text {null } \$ 4}$ (lane 3)). (B-E) Immunostaining of cells in earlier spermatocyte cysts (B, C) or in later stage cysts (D-E) with the anti-dLarp antibody. The immunostaining of dLarp is shown in red in B and C. Its single presentation appears in D'. Mitochondria were simultaneously stained with Mitofluor Red and visualized in green. The Hub cells at the tip of testis contain the highest amount of mitochondria. A co-localization of dLarp with mitochondria is represented in yellow as an overlapping of two colors (B). (Inset in B or C) A magnified view of S2 stage spermatocytes. Note that dLarp (red in inset C) is co-localized with mitochondria aggregates (green in inset B). DNA stained with DAPI is visualized in blue. Bars: $10 \mu \mathrm{m}$.

The homologies mainly fell into the La domain and conserved region (residue 818 to 1217), in which tandem repeats of the DM15 motif were contained. Although the function of these domains is still unknown, their important role in the family proteins can be inferred from their significant conservation (Fig. 5C).

\section{Distinctive co-localization of the dLarp with mitochondria aggregates was detected in early spermatocytes and meiotic cells}

To understand the intracellular localization of dLarp during spermatogenesis, we carried out immunostaining experiments using specific antibodies. We detected the immunostaining signal in the cytoplasm from germ line stem cells to spermatids at post-meiotic onion stage. The most distinct signal can be observed in spermatocytes at earlier growth stages ( $\mathrm{S} 1$ to $\mathrm{S} 2$ stages). We confirmed that these signals decreased to a background level in testes from the null type mutants (data not shown). To further confirm this immuno- localization, we examined the localization of GFP-dLarp protein expressing in a protein trap line, ZCL2496 (Kelso et al., 2004). The coincidence of the immunostaining signal with GFP-dLarp localization was confirmed throughout developmental stages in testis. An accumulation of the dLarp was initially observed in germline stem cells, although the Hub cells that are of somatic origin did not contain any detectable amount of the dLarp (Fig. 6B). The dLarp signal became more distinctive as the spermatocytes developed. In the S2 stage, mitochondria assembled to construct an aggregate at one side of each apolar cell. The distinct dLarp localization was associated with the mitochondria aggregates rather than the whole cytoplasm (Fig. $6 \mathrm{C}$, inset). However, we could not find any remarkable abnormality even in the null mutants. At that point, the dLarp was spread throughout cytoplasm at S3 stage. After S4 stage, the signal was significantly reduced (Fig. 6D). A considerable amount of the dLarp, however, reappeared just before initiation of meiosis I. There is a tendency to accumulate the dLarp as cell divisions become more active in 

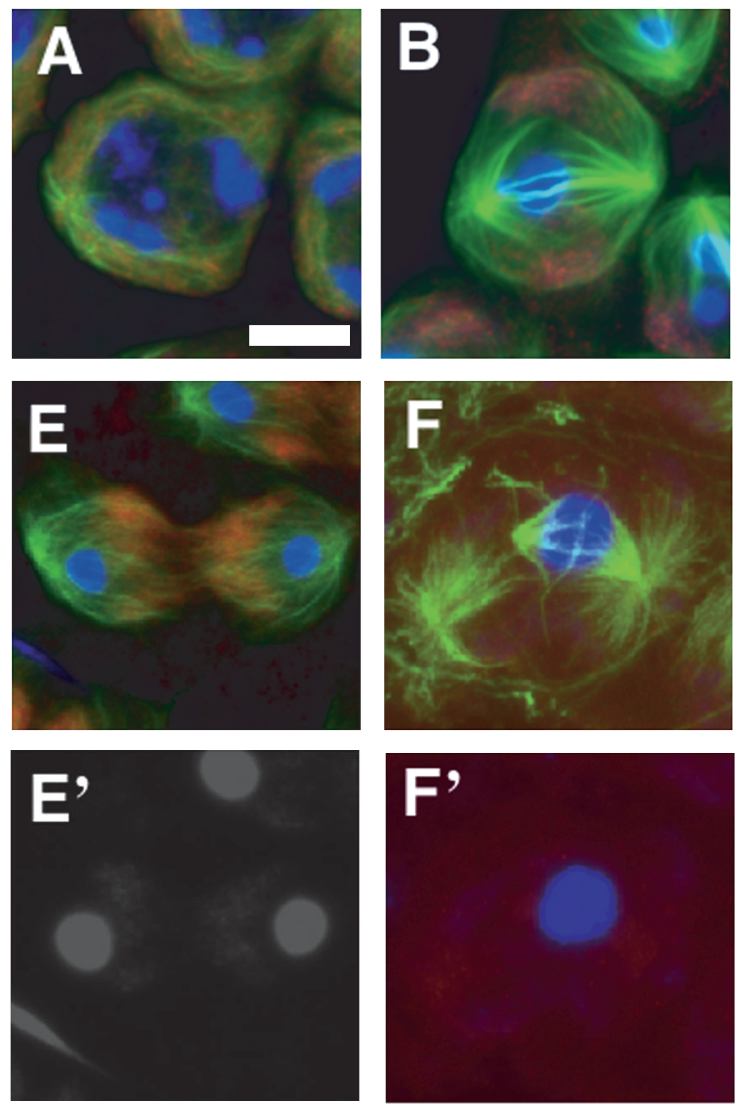
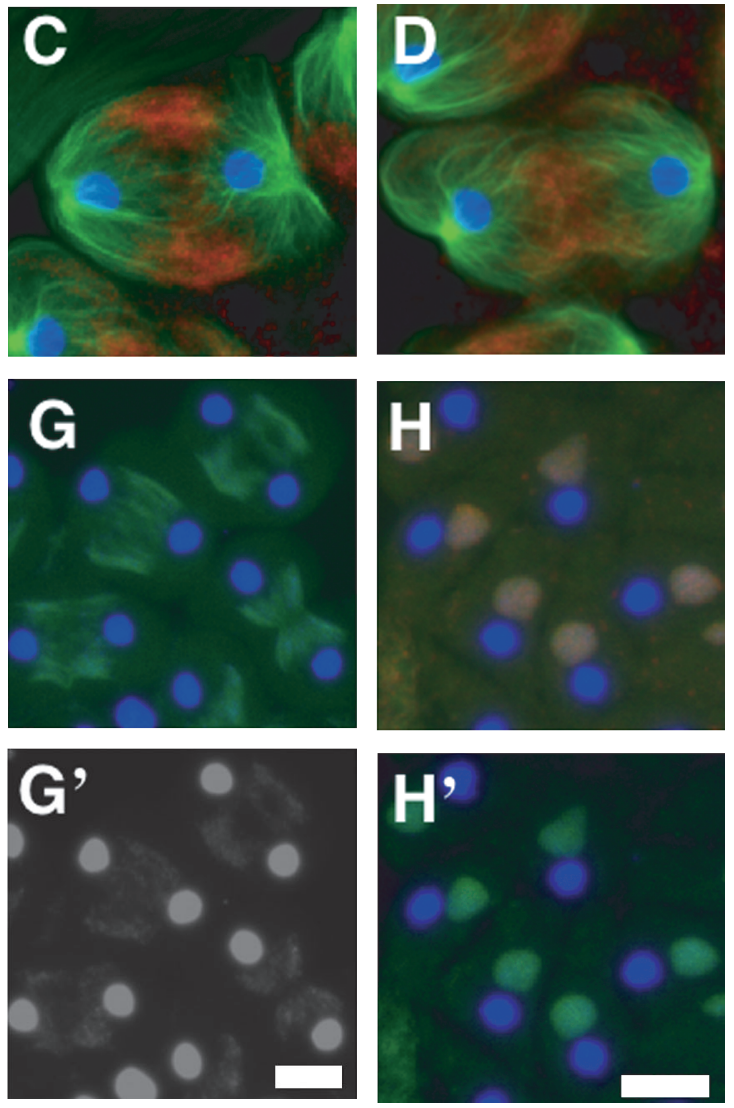

Fig. 7. The dLarp protein exhibits a close association with mitochondria during male meiotic divisions. (A-F) Immunostaining of spermatocytes undergoing meiosis I with the dLarp antibody (red). Microtubules and chromosomes are visualized in green and blue, respectively. (G) A localization of GFP-dLarp (green) is also associated with mitochondria (weaker DAPI staining in G') during meiotic division II. (H) A detection of the dLarp in spermatids at onion stage (red). (E', G') A single presentation for DAPI staining to simultaneously visualize chromosomes (stronger signal) and mitochondria (weaker signal). (F') The immunostaining signals that appeared in normal spermatocytes can no longer be detected in spermatocytes from null type mutant, larp ${ }^{43}$. $\left(\mathrm{H}^{\prime}\right)$ Nebenkerns, mitochondria derivatives are visualized by expression of EYFP-mitochondria in green. Bars: $10 \mu \mathrm{m}$.

male germ cells. The dLarp localization was concomitant with the mitochondria distribution during meiotic divisions (Fig. 7A-E). After completion of meiosis II, a weak signal could still be detected on the Nebenkerns. However, as spermatids began elongating, it faded away.

\section{Discussion}

In the present study, we isolated novel mutations for the larp gene encoding a Drosophila orthologue of a protein family with a domain exhibiting outstanding homology with a La type RNA binding protein. The larp mutant males exhibited multiple meiotic phenotypes such as defects in chromosome segregation, a failure of cytokinesis, and unequal mitochondrial partition. We show here that mitochondria as well as chromosomes inherit a mode closely linked with microtubules during Drosophila male meiosis. We observed several abnormalities in meiotic apparatus such as defects in spindle pole organization and spindle formation in the larp males. The dLarp protein is prominent in proliferating cells in testis and it particularly exhibits a distinct association with mitochondria in male meiosis.

\section{Active mechanism to ensure equal partition of mitochondria in Drosophila male meiosis}

It is well known that the highly ordered mechanism based on the spindle microtubules exists to ensure accurate chromosome segregation and cytokinesis. By contrast, it was believed that mitochondria inheritance was achieved passively as a consequence of equal cytokinesis (Shima and Warren, 1998). However, the inheritance of the cell organelles is performed in rather an active mode mediated by microtubules in fission yeast (Yaffe et al., 2003). We showed in this study that the mitochondria inheritance in Drosophila male meiosis is closely associated with aster and central spindle microtubules. They seem to be trans- 
ported along aster microtubules in plus-ended direction by metaphase. The mutant's phenotypes tell us that dLarp plays an essential role in this process, possibly working together with a plus-ended motor. As the Klp67A is known to be a functional Drosophila homologue of KIF1B that is a microtubule-based motor to transport mitochondria, this would be one of candidates involved in the transport of mitochondria (Pereira et al., 1997). The transition of mitochondria is thus regulated by an ordered mode rather than a stochastic partitioning strategy. It is possible to speculate that the dLarp is required for the active inheritance of mitochondria along microtubules in Drosophila male meiosis.

\section{Roles of dLarp in meiotic divisions in Drosophila males}

As the most characteristic phenotype of the larp mutants, we mention their multiple meiotic phenotypes. We have seen a loss of spindle pole integrity or a dysfunction of a microtubule organization center in the mutants. Such defects in cell division apparatuses could be interpreted to mean the perturbation of chromosome segregation in the larp mutants. Further, cytokinesis during meiotic divisions often fails in the larp mutants. As the central spindle microtubules are essential for initiation and progression of cytokinesis (Giansanti et al., 1998; Inoue et al., 2004), a loss of the spindle microtubules observed in the mutants would result in a failure of cytokinesis. Furthermore, the abnormal mitochondria partition would also result from a defect in microtubule structure in the mutants. It is reasonable to speculate that the dLarp may play an essential role in the formation and/or function of meiotic spindle microtubules. Therefore, a loss of the dLarp would result in multiple meiotic phenotypes highly dependent on meiotic spindle microtubules.

It is also possible to interpret the data to mean that dLarp is required for mitochondria distribution as well as for meiotic spindle formation. The dLarp may, for example, work as an anchoring protein that links mitochondria to microtubules. The Des-1 protein is an example of this case (Basu and $\mathrm{Li}, 1998$ ). This protein is supposed to be localized in the outer membrane of mitochondria and its mutant showed defects in central spindle formation. If this were also the case with the dLarp, it is possible to conjecture that a loss of the dLarp would disrupt mitochondria distribution along microtubules and would also influence microtubule structure at meiosis. It would be interesting to examine a genetic interaction between these two genes in order to test whether these proteins work as a part of anchoring components.

The dLarp may have a more specific role in mitochondria distribution. Alternatively, it might be involved in mitochondria biogenesis or morphogenesis. The larp mutations might thus prevent mitochondria from proper segregation. In these cases, it is possible to conjecture that an alteration of intrinsic mitochondria distribution affects a local ATP concentration in spermatocytes. As a secondary defect of their altered distribution, it would lead to a reduced ATPdependent activity of certain motor proteins essential for cell division. Discrete motor proteins depending on ATP hydrolysis mediate an activity to focus the minus ends of spindle microtubules and to tether the minus ends to spindle poles. It has indeed been demonstrated that mutations for the ATPase $\varepsilon$ subunit in Drosophila actually affect mitotic spindle orientation (Kidd et al., 2005).

Another possible interpretation would be that the dLarp might be involved in a more general cellular function such as a translational control of mRNAs expressed in spermatocytes before meiosis. The bristle phenotype of larp mutants is reminiscent of the Minute phenotypes caused by an insufficient ribosome biogenesis. The dLarp has a domain with remarkable homology to the La protein known as a RNA binding protein to control protein synthesis (Gottlieb and Steitz, 1989; Alfono et al., 2004). We especially noticed one larp phenotype that had a subset of spindle microtubules that was detached from spindle poles and oriented in random directions. A quite similar meiotic phenotype has also been observed in mutants for a gene encoding a poly A-binding protein (Flybase http://flybase.net/bin/fbidq. html?Fbgn0003031, Ichihara et al., in preparation). The Elf gene encoding the Efla-like protein, inferred to be a translation terminator, is another example whose mutants exhibit perturbation of chromosome segregation and cytokinesis in male meiosis as well as the generation of misshapen Nebenkerns (Basu et al., 1998). The curious similarity of meiotic phenotypes between these three proteins allows us to speculate that the dLarp is also involved in translational regulation specific to spermatogenesis. Many of the proteins required for meiotic progression and later spermatogenesis have to express and accumulate in the spermatocytes before initiation of meiotic division. Evidence that a considerable number of genes essential for the progression of this stage are involved in the translation control implies its importance in spermatogenesis (White-Cooper et al., 1998). Further examination of the larp may provide us with a clue to reveal a novel mechanism of regulation for initiation of meiotic divisions.

\section{dLarp functions in pre-meiotic cells or in somatic cells}

Although accumulation of dLarp was also found in germline stem cells, spermatogonia and S1 to S2 stage primary spermatocytes, we have not observed distinct abnormalities in these cells. There are two possible interpretations. First, other redundant proteins might compensate for the dLarp function in these germline cells. Second, the dLarp activity may be particularly indispensable for preparation of meiotic divisions. The spermatocytes undergo marked cell growth before meiosis. The phenotypes may appear more severely in enlarged spermatocytes rather than in spermatogonia. This has been often seen in other cell division mutants (Casal et al., 1990; Giansanti et al., 1998). 
Although distinct somatic phenotypes or reduced viability of the null type larp mutants have not been identified, several lines of evidence suggest its possible role in somatic cells. We confirmed that the larp is expressed at every developmental stage. The larp mutants exhibited maternal phenotypes that syncytial stage embryos from the mutant females showed abnormal progression of nuclear division cycles. We have observed accumulation of dLarp in proliferating tissues such as imaginal discs or larval brains (Inoue et al., unpublished). The larp has been identified as one of genes induced in Ras-mediated over proliferation of hemocytes in Drosophila (Asha et al., 2003). These results indicate its possible role in cell proliferation of somatic cells. The larp was originally described as a possible target of $S c r$ or $U b x$ in embryogenesis (Chauvet et al., 2000), hence the protein may be involved in a cellular function in response to morphogenetic signals. Absence of significant somatic phenotypes allows us to consider the existence of redundant proteins to compensate for dLarp in somatic cells. In order to identify genes that interact with the larp, a large genetic screening of enhancer mutations for the larp is now underway.

Acknowledgements. We wish to thank the Kyoto Drosophila Genetic Resource Center for providing fly stocks. We also wish to acknowledge Drs. Melanie Gatt and David Glover for sharing some of their unpublished results and for a gift of larp $^{\text {null }}{ }^{4}$. We wish to thank Drs. Thomas Kaufman and Ernst Wimmer for their gifts of anti-CNN antibody and larp ${ }^{43}$, respectively. This work was supported in part by Grants-in-Aid for Scientific Research on Priority Area from the Ministry of Education, Culture, Sports, Science and Technology of Japan.

\section{References}

Alfano, C., Sanfelice, D., Babon, J., Kelly, G., Jacks, A., Curry, S., and Conte, M.R. 2004. Structural analysis of cooperative RNA binding by the La motif and central RRM domain of human La protein. Nat. Struct. Mol. Biol., 11: 323-329.

Arama, E., Bader, M., Srivastava, M., Bergmann, A., and Steller, H. 2006. The two Drosophila cytochrome C proteins can function in both respiration and caspase activation. EMBO J., 25: 232-243.

Asha, H., Nagy, I., Kovacs, G., Stetson, D., Ando, I., and Dearolf, C.R. 2003. Analysis of Ras-induced overproliferation in Drosophila hemocytes. Genetics, 163: 203-215.

Basu, J. and Li, Z. 1998. The Des-1 protein, required for central spindle assembly and cytokinesis, is associated with mitochondria along the meiotic spindle apparatus and with the contractile ring during male meiosis in Drosophila melanogaster. Mol. Gen. Genet., 259: 664-673.

Basu, J., Williams, B.C., Li, Z., Williams, E.V., and Goldberg, M.L. 1998. Depletion of a Drosophila homolog of yeast Sup35p disrupts spindle assembly, chromosome segregation, and cytokinesis during male meiosis. Cell Motil. Cytoskeleton, 39: 286-302.

Bonaccorsi, S., Giansanti, M.G., and Gatti, M. 1998. Spindle self-organization and cytokinesis during male meiosis in asterless mutants of Drosophila melanogaster. J. Cell Biol., 142: 751-761.

Casal, J., Gonzalez, C., Wandosell, F., Avila, J., and Ripoll, P. 1990. Abnormal meiotic spindles cause a cascade of defects during spermatogenesis in asp males of Drosophila. Development, 108: 251-260.

Castrillon, D.H., Gonczy, P., Alexander, S., Rawson, R., Eberhart, C.G., Viswanathan, S., DiNardo, S., and Wasserman, S.A. 1993. Toward a molecular genetic analysis of spermatogenesis in Drosophila melano- gaster: characterization of male-sterile mutants generated by single $\mathrm{P}$ element mutagenesis. Genetics, 135: 489-505.

Cenci, G., Bonaccorsi, S., Pisano, C., Verni, F., and Gatti, M. 1994. Chromatin and microtubule organization during premeiotic, meiotic and early postmeiotic stages of Drosophila melanogaster spermatogenesis. J. Cell Sci., 107: 3521-3534.

Chauvet, S., Maurel-Zaffran, C., Miassod, R., Jullien, N., Pradel, J., and Aragnol, D. 2000. dlarp, a new candidate Hox target in Drosophila whose orthologue in mouse is expressed at sites of epithelium/mesenchymal interactions. Dev. Dyn., 218: 401-413.

Deak, P., Omar, M.M., Saunders, R.D., Pal, M., Komonyi, O., Szidonya, J., Maroy, P., Zhang, Y., Ashburner, M., Benos, P., Savakis, C., SidenKiamos, I., Louis, C., Bolshakov, V.N., Kafatos, F.C., Madueno, E., Modolell, J., and Glover, D.M. 1997. P-element insertion alleles of essential genes on the third chromosome of Drosophila melanogaster: correlation of physical and cytogenetic maps in chromosomal region 86E-87F. Genetics, 147: 1697-1722.

Eggert, U.S., Mitchison, T.J., and Field, C.M. 2006. Animal cytokinesis: from parts list to mechanisms. Annu. Rev. Biochem., 75: 543-566.

Fuller, M.T., Caulton, J.H., Hutchens, J.A., Kaufman, T.C., and Raff, E.C. 1988. Mutations that encode partially functional beta 2 tubulin subunits have different effects on structurally different microtubule arrays. J. Cell Biol., 107: 141-152.

Fuller, M.T. 1993. Spermatogenesis. In The development of Drosophila. (M. Bate and A. Martinez-Arias eds.) Cold Harbor Laboratory Press, Cold Spring Harbor, New York, pp.71-147.

Giansanti, M.G., Bonaccorsi, S., Williams, B., Williams, E.V., Santolamazza, C., Goldberg, M.L., and Gatti, M. 1998. Cooperative interactions between the central spindle and the contractile ring during Drosophila cytokinesis. Genes Dev., 12: 396-410.

Gottlieb, E. and Steitz, J.A. 1989. Function of the mammalia La protein: evidence for its action in transcription termination by RNA polymerase III. EMBO J., 8: 851-861.

Horn, C., Offen, N., Nystedt, S., Hacker, U., and Wimmer, E.A. 2003. piggyBac-based insertional mutagenesis and enhancer detection as a tool for functional insect genomics. Genetics, 163: 647-661.

Inoue, Y.H., do Carmo Avides, M., Shiraki, M., Deak, P., Yamaguchi, M., Nishimoto, Y., Matsukage, A., and Glover, D.M. 2000. Orbit, a novel microtubule-associated protein essential for mitosis in Drosophila melanogaster. J. Cell Biol., 149: 153-166.

Inoue, Y.H., Savoian, M.S., Suzuki, T., Mathe, E. Yamamoto, M.T., and Glover, D.M. 2004. Mutations in orbit/mast reveal that the central spindle is comprised of two microtubule populations, those that initiate cleavage and those that propagate furrow ingression. J. Cell Biol., 166: 49-60.

Kelso, R.J., Buszczak, M., Quinones, A.T., Castiblanco, C., Mazzalupo, S., and Cooley, L. 2004. Flytrap, a database documenting a GFP proteintrap insertion screen in Drosophila melanogaster. Nucleic. Acid. Res., 32: D418-D420.

Kidd, T., Abu-Shumays, R., Katzen, A., Sisson, J.C., Jimenez, G., Pinchin, S. Sullivan, W., Ish-Horowicz, D. 2005. The epsilon-subunit of mitochondrial ATP synthase is required for normal spindle orientation during the Drosophila embryonic divisions. Genetics, 170: 697-708.

Kiefel, B.R., Gilson, P.R., and Beech, P.L. 2006. Cell biology of mitochondrial dynamics. Int. Rev. Cytol., 254: 151-213.

Li, K. and Kaufman, T.C. 1995. The homeotic target gene centrosomin encodes an essential centrosomal component. Cell, 85: 585-596.

Maraia, R.J. and Intine, R.V. 2001. Recognition of nascent RNA by the huma La antigen: conserved and divergent features of structure and function. Mol. Cell Biol., 21: 367-379.

Nangaku, M., Sato-Yoshitake, R., Okada, Y., Noda, Y., Takemura, R., Yamazaki, H., and Hirokawa, N. 1994. KIF1B, a novel microtubule plus end-directed monomeric motor protein for transport of mitochon- 
dria. Cell, 79: 1209-1220.

Pereira, A.J., Dalby, B., Stewart, R.J., Doxsey, S.J., Goldstein, L.S.B 1997. Mitochondria association of a plus end-directed microtubule motor expressed during mitosis in Drosophila. J. Cell Biol., 136: 10811090.

Ponting, C.P., Mott, R., Bork, P., and Copley, R.R. 2001. Novel protein domains and repeats in Drosophila melanogaster: insights into structure, function, and evolution. Genome Res., 11: 1996-2008.

Shima, D.T. and Warren, G. 1998. Inheritance of the cytoplasm during cell division. In Dynamics of cell division. (S.A. Endow and D.M. Glover eds.) Oxford University Press, Oxford, pp.248-269.

Vernos, I. and Karsenti, E. 1998. Microtubule dynamics, molecular motors, and chromosome behavior. In Dynamics of cell division. (S.A. Endow and D.M. Glover eds.) Oxford Press, Oxford, pp.97-123.

White-Cooper, H., Schafer, M.A., Alphey, L., and Fuller, M.T. 1998. Transcriptional and post-transcriptional control mechanisms coordinate the onset of spermatid differentiation with meiosis I in Drosophila. Development, 125: 125-134.

Yaffe, M.P., Stuurman, N., and Vale, R.D. 2003. Mitochondrial positioning in fission yeast is driven by association with dynamic microtubules and mitotic spindle poles. Proc. Natl. Acad. Sci. USA., 100: 1142411428 .

(Received for publication, July 26, 2007 and accepted, September 10, 2007) 